\title{
Parameter Optimization and Sensitivity Studies of Spontaneous Fission with FREYA
}

\author{
R. Vogt ${ }^{1,2}$, J. Randrup ${ }^{3}$, P. Talou ${ }^{4}$, J. T. Van Dyke ${ }^{5}$ and L. A. Bernstein ${ }^{3,6}$ \\ ${ }^{1}$ Nuclear and Chemical Sciences Division, Lawrence Livermore National Laboratory, Livermore, CA 94551, USA \\ e-mail: vogt2@1lnl.gov \\ ${ }^{2}$ Physics Department, University of California at Davis, Davis, CA 95616, USA \\ ${ }^{3}$ Nuclear Science Division, Lawrence Berkeley National Laboratory, Berkeley, CA 94720, USA \\ ${ }^{4}$ Theoretical Division, Los Alamos National Laboratory, Los Alamos, NM 87545, USA \\ ${ }^{5}$ Physics Department, University of California at Berkeley, Berkeley, CA 94720, USA \\ ${ }^{6}$ Nuclear Engineering Department, University of California at Berkeley, Berkeley, CA 94720, USA
}

\begin{abstract}
For many years, the state of the art for simulating fission in transport codes amounted to sampling from average distributions. However, such "average" fission models have limited capabilities. Energy is not explicitly conserved and no correlations are available because all particles are emitted independently. However, in a true fission event, the emitted particles are correlated. Recently, Monte Carlo codes generating complete fission events have been developed, thus allowing the use of event-by-event analysis techniques. Such techniques are particularly useful because the complete kinematic information is available for the fission products and the emitted neutrons and photons. It is therefore possible to extract any desired observables, including correlations. The fast event-by-event fission code FREYA (Fission Reaction Event Yield Algorithm) generates large samples of complete fission events, employing only a few physics-based parameters. A recent optimization of these parameters for the isotopes in FREYA that undergo spontaneous fission is described and results are presented. The sensitivity of neutron observables in FREYA to the input yield functions is also discussed and the correlation between the average neutron multiplicity and fragment total kinetic energy is quantified.
\end{abstract}

\section{Introduction}

In this proceedings, we introduce and describe the fission simulation code FREYA [1, 2]. The input parameters and their physical effects are presented. Then the results of the parameter optimization for spontaneous fission in FREYA [5] is discussed. A study of the sensitivity of the FREYA results for ${ }^{252} \mathrm{Cf}(\mathrm{sf})$ to the input yields is described and the resulting correlation between neutron multiplicity $\bar{v}$ and fragment total kinetic energy is shown [6].

\section{Brief Description of FREYA}

As input, FREYA requires the mass distribution of the primary fission fragments, $Y(A)$, and the mean total kinetic energy for a given mass split, $\overline{\operatorname{TKE}}(A)$, for the particular excitation considered. (FREYA can simulate both neutroninduced fission and spontaneous fission.) As we discuss later, FREYA can also be modified to take inputs in the form $Y(A, Z, \mathrm{TKE})$.

The initial nucleus $A_{0}$ splits into light and heavy fragments, $A_{L}$ and $A_{H}$ respectively. The $Q$-value for a particular split is $Q=M_{0} c^{2}-M_{L} c^{2}-M_{H} c^{2}$. The total fragment kinetic energy, TKE, is sampled from $\operatorname{TKE}\left(A_{H}\right)$ and the total excitation energy available for rotational and statistical excitation at scission is $E_{\mathrm{sc}}^{*}=Q-\overline{\mathrm{TKE}}$. The corresponding 'scission temperature' $T_{\mathrm{sc}}$ is obtained from $E_{\mathrm{sc}}^{*}=a\left(A_{0}\right) T_{\mathrm{sc}}^{2}$ where the scale of the level density parameter $a(A)=A / e_{0}$ is governed by $e_{0} \approx 10 \mathrm{MeV}$. This is the first adjustable parameter in FREYA. The parameter $e_{0}$ only has a direct effect on the prompt fission neutron spectrum (PFNS).

In addition to any overall rigid rotation, which imparts mean angular momenta to the two fragments, they also acquire fluctuations around the mean values from the wriggling and bending modes. The magnitude of these spin fluctuations is governed by the 'spin temperature' $T_{S}=c_{S} T_{\text {sc }}$ which can be adjusted through the second FREYA parameter $c_{S}$ [3]. The spin fluctuations vanish for $c_{S}=0$. Since $c_{S}$ determines the amount of rotational energy, it is the only parameter that has a direct influence on photon observables. It only has an indirect effect on neutron observables through its influence on the partition of the excitation energy between intrinsic excitation and rotational energy of the fragments.

After subtracting the rotational energy of the two fragments, $E_{\mathrm{rot}}$, a total of $E_{\mathrm{stat}}=E_{\mathrm{sc}}^{*}-E_{\mathrm{rot}}$ is left for statistical excitation which is distributed between the two fragments. A preliminary partition, $E_{\text {stat }}=E_{L}^{*}+E_{H}^{*}$, is made according to the heat capacities of the fragments, which in turn is assumed to be proportional to the level density parameters, i.e. $\hat{E}_{L}^{*}: \hat{E}_{H}^{*}=a_{L}: a_{H}$. If the shell corrections are negligible, or the available energy is large, $a_{i} \approx A_{i} / e_{0}$. Because the observed neutron multiplicities for known nu- 
clei at low energies suggest that the light fragments tend to be disproportionately excited, the light fragment is given a larger excitation energy by the third parameter $x$,

$$
\begin{aligned}
\bar{E}_{L}^{*} & =x \hat{E}_{L}^{*} \\
\bar{E}_{H}^{*} & =E_{\text {stat }}-\bar{E}_{L}^{*}
\end{aligned}
$$

where $x>1$ [4]. We discuss the consequences of a massdependent $x$ parameter in a later section. The value of $x$ strongly influences $v(A)$ and the neutron-neutron correlations.

After the mean fragment excitation energies have been assigned, FREYA considers thermal fluctuations in the statistical excitation. The mean fragment excitation is related to its temperature $T_{i}$ by $\bar{E}_{i}^{*}=a_{i} T_{i}^{2}$ with associated variance $\sigma_{E_{i}}^{2}=2 \bar{E}_{i}^{*} T_{i}$. An energy fluctuation $\delta E_{i}^{*}$ is sampled from a truncated normal distribution of variance $2 c \bar{E}_{i}^{*} T_{i}$ and the fragment excitations are adjusted accordingly,

$$
E_{i}^{*}=\bar{E}_{i}^{*}+\delta E_{i}^{*}, i=L, H .
$$

Energy is conserved by making a compensating opposing fluctuation in TKE,

$$
\mathrm{TKE}=\overline{\mathrm{TKE}}-\delta E_{L}^{*}-\delta E_{H}^{*} .
$$

The factor $c$ multiplying the variance is the fourth FREYA parameter; it compensates for the truncation of the normal distribution due to energy conservation and directly affects the shape and moments of $P(v)$. Finally, TKE may be adjusted by the fifth and final FREYA parameter, $d$ TKE, to reproduce the average neutron multiplicity, $\bar{v}$.

The neutrons are evaporated isotropically in the frame of the emitting fragment, apart from a slight flattening due to the nuclear rotation. Their energy is sampled from a black-body energy spectrum, $d N_{n} / d E_{n} \sim$ $E_{n} \exp \left(-E_{n} / T_{\max }\right)$, where $T_{\max }$ is the maximum possible temperature in the daughter nucleus. FREYA generally assumes that neutron evaporation continues until the nuclear excitation energy is below the neutron separation energy $S_{\mathrm{n}}$, so that neutron evaporation continues as long as energetically possible. Note that while certain parameters have a direct effect on a particular observables, all can affect the shape of the PFNS, thus it is sensitive to all the parameters.

After neutron evaporation has ceased, the excited product nucleus emits photons. First, statistical photons are emitted isotropically with an energy distribution sampled from a black-body spectrum modulated by a giant-dipole resonance form factor. When the nuclear excitation energy enters the regime of the tabulated decays from the RIPL3 compilation [7], FREYA switches to a discrete cascade which is continued until the half-life exceeds a specified value, $t_{\max }$, based on the detector response time, or until the nucleus is in its ground state. The average photon energy and photon multiplicity depends on $t_{\max }$ as well as the minimum photon energy measurable in the detectors, denoted in FREYA as $g_{\text {min }}$. While the photon observables depend on both $t_{\max }$ and $g_{\min }$, these quantities are not parameters but are external to FREYA.

\section{Parameter optimization for spontaneous fission}

The five FREYA parameters, $e_{0}, c_{S}, x, c$ and $d \mathrm{TKE}$, are all physics-based. As noted in Sec. 2, a particular parameter can affect some observables directly without having any significant effect on others.

A first attempt to make a global fit of the five FREYA parameters for ${ }^{252} \mathrm{Cf}(\mathrm{sf})$ was made in Ref. [8] using a grid search method. More recently an optimization using simulated annealing was able to generally reproduce these results as well as provide variances and covariances on the parameter values [5]. Similar studies were carried out for all spontaneous fission isotopes in FREYA.

Optimized input parameters were determined for FREYA [9] by means of simulated annealing [5]. For each possible set of parameter values, a "cost" is assigned given by the $\chi^{2}$ value associated with the resulting deviation of the FREYA results from the considered experimental data. A Metropolis-type random walk in the five-dimensional parameter space is then performed while gradually reducing the associated "temperature", repeating this annealing process several times to prevent the system from getting trapped in local minima. In this manner, one may obtain not only the optimal values of the five FREYA parameters but also their mutual correlations, as expressed by the covariance matrix evaluated at the optimal site.

The results for all isotopes undergoing spontaneous fission in FREYA are given in Table 1. Note that, in some cases, only a single data set or evaluation is available for optimization. In general, however, this single set is an evaluation of the neutron multiplicity distribution and its first three moments, providing multiple observables. However, these observables are only directly associated with the parameter $c$, affecting the width of $P(v)$, and indirectly to $d$ TKE through the value of the neutron multiplicity $\bar{v}$. The results for the first three neutron multiplicity moments are given in Table 2 while the average photon multiplicity $\bar{N}_{\gamma}$ and average energy per photon $\bar{\epsilon}_{\gamma}$ are shown in Table 3 for all the isotopes considered. The results are compared to data where applicable: in particular, photon data are not available for all isotopes. The FREYA uncertainties are influenced by the uncertainties on the parameter values and are not related to statistical uncertainties.

Some general trends in the results can be observed for all the cases studied. In particular, the asymptotic value of the level density parameter $e_{0}$, the excitation energy sharing parameter $x$, and the scale factor of the scission temperature setting the photon energy and multiplicity $c_{S}$ remain unchanged within the uncertainties, namely $\left\langle e_{0}\right\rangle \approx 10.2 / \mathrm{MeV},\langle x\rangle \approx 1.2$ and $\left\langle c_{S}\right\rangle \approx 0.89$ respectively. Thus these quantities are rather isotope independent. One might expect that $e_{0}$, regulating the temperature of the fragment, as well as that of the spontaneously fissioning nucleus itself, for neutron emission has a universal value. However, it is the least constrained of all the parameters because no observables are directly sensitive to $e_{0}$ except the prompt fission neutron spectrum which is sensitive to all five parameters. The parameters $c$ and $d \mathrm{TKE}$ are strongly isotope dependent. In some cases, where $c$ 
Table 1. Results of the optimization for all spontaneously fissioning isotopes which are modeled by FREYA. The best fit values of the five parameters and their associated variances are given for each isotope. In addition, the number of data sets and evaluations used for each isotope are indicated. The evaluations of Ref. [10], available for all isotopes, provides multiple observables: the neutron multiplicity distribution $P(v)$ and its first three moments: $\bar{v}, v_{2}$ and $v_{3}$. From Ref. [5].

\begin{tabular}{|c|c|c|c|c|c|c|}
\hline Parameter & ${ }^{238} \mathrm{U}(\mathrm{sf})$ & ${ }^{238} \mathrm{Pu}(\mathrm{sf})$ & ${ }^{240} \mathrm{Pu}(\mathrm{sf})$ & ${ }^{242} \mathrm{Pu}(\mathrm{sf})$ & ${ }^{244} \mathrm{Cm}(\mathrm{sf})$ & ${ }^{252} \mathrm{Cf}(\mathrm{sf})$ \\
\hline$e_{0}$ & $10.39 \pm 0.12$ & $10.52 \pm 0.34$ & $10.75 \pm 0.02$ & $10.02 \pm 3.24$ & $10.49 \pm 2.31$ & $10.43 \pm 1.19$ \\
\hline$x$ & $1.22 \pm 0.01$ & $1.23 \pm 0.04$ & $1.31 \pm 0.01$ & $1.14 \pm 0.02$ & $1.24 \pm 0.02$ & $1.27 \pm 0.04$ \\
\hline$c$ & $0.94 \pm 0.08$ & $1.97 \pm 0.01$ & $3.18 \pm 0.13$ & $3.42 \pm 0.12$ & $1.39 \pm 0.34$ & $1.19 \pm 0.13$ \\
\hline$c_{S}$ & $0.90 \pm 0.08$ & $0.89 \pm 0.01$ & 0.91 & $0.91 \pm 0.07$ & $0.91 \pm 0.10$ & $0.88 \pm 0.04$ \\
\hline$d$ TKE & $-1.38 \pm 0.53$ & $-1.41 \pm 17.68$ & $-3.22 \pm 0.02$ & $-1.66 \pm 0.01$ & $-4.49 \pm 0.023$ & 0.52 \\
\hline \# Sets & 1 & 1 & 2 & 3 & 3 & 6 \\
\hline
\end{tabular}

is large, the neutron multiplicity is close to 2 and FREYA would produce a rather narrow $P(v)$ without increasing $c$. Interestingly, the multiplicity distribution for ${ }^{238} \mathrm{U}(\mathrm{sf})$ is so narrow that it forces $c<1$ in this case. The values of $d \mathrm{TKE}$ are strongly correlated with $c$ (positive correlation) as well as $e_{0}$ and $c_{S}$ (negative correlation) because these parameters are more directly related to $\bar{v}$ than $x$ is since changing $x$ can change $v(A)$ while keeping $\bar{v}$ fixed. See Ref. [5] for more details and for comparison to data. This study is being followed by parameter optimization for neutron-induced fission.

\section{Sensitivity of neutron observables in ${ }^{252} \mathbf{C f}(\mathbf{s f})$ to the input yields}

In this section, an investigation of the sensitivity of the FREYA results to the model inputs, in particular the yield functions [6]. This work sought to quantify the degree to which the measured yields can constrain $\bar{v}$. An ensemble of yield functions were obtained from available data and then run through FREYA. The effects on the neutron observables were quantified and covariances between these observables were calculated. Finally, the correlation between the variances of $\bar{v}$ and $\overline{\mathrm{TKE}}$ was derived (for details, see Ref. [6]).

A least-squares statistical analysis of available experimental data was performed on $Y(A)$ [11-16], $\overline{\operatorname{TKE}}\left(A_{H}\right)$ [11-13, 16-18], and $\sigma_{\mathrm{TKE}}\left(A_{H}\right)[11,12,16]$ for primary fission fragments from the spontaneous fission of ${ }^{252} \mathrm{Cf}$. Wahl systematics [19] were used to obtain the charge distribution for a given mass, $Y(Z \mid A)$. Most of the experimental data included only limited information on associated uncertainties. A least-squares fit to all these data provides a mean yield function $Y(A, Z$, TKE) as well as a covariance matrix that contains uncertainties and correlations for different fragment masses. To study the influence of different yield functions on FREYA simulations, calculations were made with an ensemble of up to 15,000 yield functions, $\left\{Y_{i}(Z, A, \mathrm{TKE})\right\}$, sampled from the covariance matrix obtained from the fit. (See Ref. [6] for full details.)

For each yield function $Y_{i}(A, Z, \mathrm{TKE})$, the extracted observables are assigned a weight $W_{i}$ and the corresponding weighted ensemble average values (and the associated correlations) can then be calculated. In the standard unbiased treatment, all $N$ sampled yield functions are given the same weight, $W_{i}=1 / N$. However, because the average neutron multiplicity $\bar{v}$ is known very accurately, it is interesting to "reward" a given yield function based on how well it reproduces that target value. Such a bias can be implemented by means of the weight function $W_{i} \sim \exp \left[-\left(\bar{v}^{(i)}-v_{0}\right)^{2} / 2 \sigma_{0}^{2}\right]$, where $v_{0}=3.756$ is the experimental value and $\sigma_{0}=0.015$ the associated uncertainty [20].

For each yield function, the generation of $10^{6}$ FREYA events was sufficient to ensure that the statistical fluctuations (from the finite event number) are unimportant. Ensemble average distributions, covariances, and moments of distributions can then be reliably calculated for various observables, as illustrated below for the neutron multiplicity distribution.

For a given yield function $Y_{i}(A, Z, \mathrm{TKE})$, one can extract the neutron multiplicity distribution $P_{n}^{(i)}(v)$ and the resulting ensemble-averaged distribution is then

$$
\prec P_{n}(v)>=\sum_{i=1}^{N} W_{i} P_{n}^{(i)}(v),
$$

also normalized to unity, $\sum_{v} \prec P_{n}(v)>\equiv 1$. The corresponding covariance matrix be also constructed from the ensemble of multiplicity distributions,

$$
\begin{aligned}
& C_{n n}\left(v, v^{\prime}\right) \\
= & \left\langle P_{n}(v) P_{n}\left(v^{\prime}\right)>-\prec P_{n}(v)>\prec P_{n}\left(v^{\prime}\right)>\right. \\
= & \sum_{i=1}^{N} W_{i} P_{n}^{(i)}(v) P_{n}^{(i)}\left(v^{\prime}\right)-\prec P_{n}(v)>\prec P_{n}\left(v^{\prime}\right)>.
\end{aligned}
$$

Its diagonal elements are the variances of $\left\{P_{n}^{(i)}(\mu)\right\}$,

$$
\begin{aligned}
& C_{n n}(v, v)=\sigma_{v}^{2} \\
& =\sum_{i=1}^{N} W_{i} P_{n}^{(i)}(v) P_{n}^{(i)}(v)-\left(\prec P_{n}(v) \succ\right)^{2} .
\end{aligned}
$$

The factorial moments of the multiplicity distribution $P_{n}^{(i)}(\mu)$ are given by

$$
M_{n}^{(i)} \equiv \sum_{v} v(v-1) \cdots(v-n+1) P_{v}^{(i)}(v),
$$

where $M_{0}^{(i)}=1$ and $M_{1}^{(i)}=\bar{v}$.

One of the main results of this work is the quantification of the relation between $v$ and TKE. These observables 
Table 2. The values of the neutron multiplicity moments, $v_{1}=\bar{v}, v_{2}$ and $v_{3}$ from the evaluation in Ref. [10], calculated by FREYA, and the ratio $\mathrm{C} / \mathrm{E}$ (calculation to evaluation). Note that the FREYA uncertainties are not statistical but represent the uncertainties on the parameters. The FREYA uncertainties increase for the higher moments because the neutron multiplicity is multiplied for $v_{2}$ and $v_{3}$.

From Ref. [5].

\begin{tabular}{|c|c|c|c|c|c|c|c|}
\hline$\overline{v_{i}}$ & Evaluation & FREYA & $\mathrm{C} / \mathrm{E}$ & $v_{i}$ & Evaluation & FREYA & $\mathrm{C} / \mathrm{E}$ \\
\hline \multicolumn{4}{|c|}{${ }^{238} \mathrm{U}(\mathrm{sf})$} & \multicolumn{4}{|c|}{${ }^{242} \mathrm{Pu}(\mathrm{sf})$} \\
\hline $\bar{v}$ & $1.98 \pm 0.03$ & $2.00 \pm 0.94$ & $1.01 \pm 0.22$ & $\bar{v}$ & $2.15 \pm 0.008$ & $2.12 \pm 1.19$ & $0.99 \pm 0.30$ \\
\hline$v_{2}$ & $2.87 \pm 0.14$ & $2.87 \pm 3.37$ & $1.00 \pm 1.37$ & $\overline{v_{2}}$ & $3.81 \pm 0.036$ & $3.79 \pm 4.51$ & $0.99 \pm 1.40$ \\
\hline$\overline{v_{3}}$ & $2.82 \pm 0.48$ & $2.83 \pm 9.81$ & $1.00 \pm 11.71$ & $v_{3}$ & $5.35 \pm 0.036$ & $5.36 \pm 12.13$ & $1.00 \pm 5.14$ \\
\hline \multicolumn{4}{|c|}{${ }^{38} \mathrm{Pu}(\mathrm{sf})$} & \multicolumn{4}{|c|}{${ }^{244} \mathrm{Cm}(\mathrm{sf})$} \\
\hline $\bar{v}$ & $2.19 \pm 0.07$ & $2.17 \pm 1.15$ & $0.99 \pm 0.27$ & $\bar{v}$ & $2.71 \pm 0.01$ & $2.70 \pm 1.16$ & $1.00 \pm 0.18$ \\
\hline$v_{2}$ & 3.87 & $3.85 \pm 4.35$ & $0.99 \pm 1.26$ & $v_{2}$ & $5.94 \pm 0.02$ & $5.95 \pm 5.46$ & $1.00 \pm 0.84$ \\
\hline$v_{3}$ & 5.42 & $5.25 \pm 10.97$ & $0.97 \pm 4.10$ & $v_{3}$ & $10.11 \pm 0.18$ & $10.17 \pm 16.78$ & $1.01 \pm 2.78$ \\
\hline \multicolumn{4}{|c|}{${ }^{240} \mathrm{Pu}(\mathrm{sf})$} & \multicolumn{4}{|c|}{${ }^{252} \mathrm{Cf}(\mathrm{sf})$} \\
\hline $\bar{v}$ & $2.15 \pm 0.005$ & $2.22 \pm 1.25$ & $1.03 \pm 0.33$ & $\bar{v}$ & $3.757 \pm 0.01$ & $3.74 \pm 1.30$ & $1.00 \pm 0.12$ \\
\hline$v_{2}$ & $3.79 \pm 0.029$ & $4.26 \pm 4.98$ & $1.12 \pm 1.66$ & $v_{2}$ & $11.95 \pm 0.02$ & $11.94 \pm 8.79$ & $1.00 \pm 0.54$ \\
\hline$v_{3}$ & $5.21 \pm 0.15$ & $6.53 \pm 13.30$ & $1.25 \pm 6.51$ & $v_{3}$ & $31.67 \pm 0.18$ & $31.84 \pm 39.94$ & $1.01 \pm 1.59$ \\
\hline
\end{tabular}

Table 3. The values of the average photon multiplicity $\bar{N}_{\gamma}$ and average energy per photon $\bar{\epsilon}_{\gamma}$ (in MeV) from data (where available), the values calculated with FREYA, and the relevant $\mathrm{C} / \mathrm{E}$ where applicable. Note that the FREYA uncertainties are not statistical but represent the uncertainties on the parameters. From Ref. [5].

\begin{tabular}{|c|c|c|c|c|c|c|c|}
\hline Average & Data & FREYA & $\mathrm{C} / \mathrm{E}$ & Average & Data & FREYA & $\mathrm{C} / \mathrm{E}$ \\
\hline \multicolumn{4}{|c|}{${ }^{238} \mathrm{U}(\mathrm{sf})$} & \multicolumn{4}{|c|}{${ }^{242} \mathrm{Pu}(\mathrm{sf})$} \\
\hline $\bar{N}_{\gamma}$ & - & $6.49 \pm 2.42$ & - & $\bar{N}_{\gamma}$ & $6.72 \pm 0.07$ & $6.61 \pm 2.43$ & $0.96 \pm 0.13$ \\
\hline$\overline{\bar{\epsilon}_{\gamma}}$ & - & $0.94 \pm 0.87$ & - & $\overline{\bar{\epsilon}_{\gamma}}$ & $0.84 \pm 0.01$ & $0.96 \pm 0.89$ & $1.14 \pm 1.12$ \\
\hline \multicolumn{4}{|c|}{${ }^{238} \mathrm{Pu}(\mathrm{sf})$} & \multicolumn{4}{|c|}{${ }^{244} \mathrm{Cm}(\mathrm{sf})$} \\
\hline $\bar{N}_{\gamma}$ & - & $6.47 \pm 2.43$ & - & $\bar{N}_{\gamma}$ & - & $7.07 \pm 2.36$ & - \\
\hline$\overline{\bar{\epsilon}_{\gamma}}$ & - & $1.05 \pm 0.93$ & - & $\overline{\bar{\epsilon}_{\gamma}}$ & - & $1.01 \pm 0.93$ & - \\
\hline \multicolumn{4}{|c|}{${ }^{240} \mathrm{Pu}(\mathrm{sf})$} & \multicolumn{4}{|c|}{${ }^{252} \mathrm{Cf}(\mathrm{sf})$} \\
\hline $\bar{N}_{\gamma}$ & $8.20 \pm 0.40$ & $6.60 \pm 2.48$ & $0.80 \pm 0.09$ & $\bar{N}_{\gamma}$ & $8.14 \pm 0.40$ & $7.71 \pm 2.80$ & $0.85 \pm 0.12$ \\
\hline $\bar{\epsilon}_{\gamma}$ & $0.80 \pm 0.07$ & $1.00 \pm 0.91$ & $1.24 \pm 1.26$ & $\bar{\epsilon}_{\gamma}$ & $0.94 \pm 0.05$ & $0.91 \pm 0.86$ & $0.97 \pm 0.83$ \\
\hline
\end{tabular}

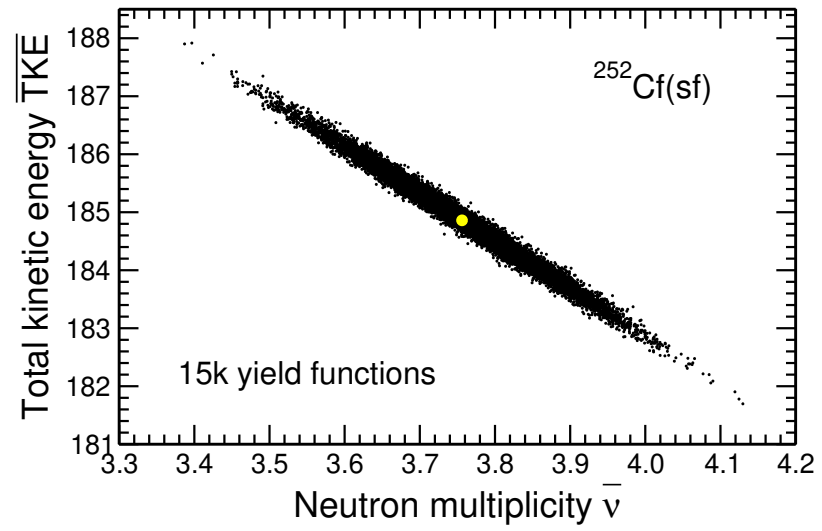

Figure 1. Scatter plot of the $\bar{v}$ and $\overline{\mathrm{TKE}}$, obtained for each of the 15,000 sampled yield functions. The overall average is indicated by the central dot. (From Ref. [6].)

exhibit a pronounced anti-correlation due to energy conservation: a larger/smaller kinetic energy is compensated by a correspondingly lower/higher total fragment excitation which in turn decreases/increases the neutron mul-

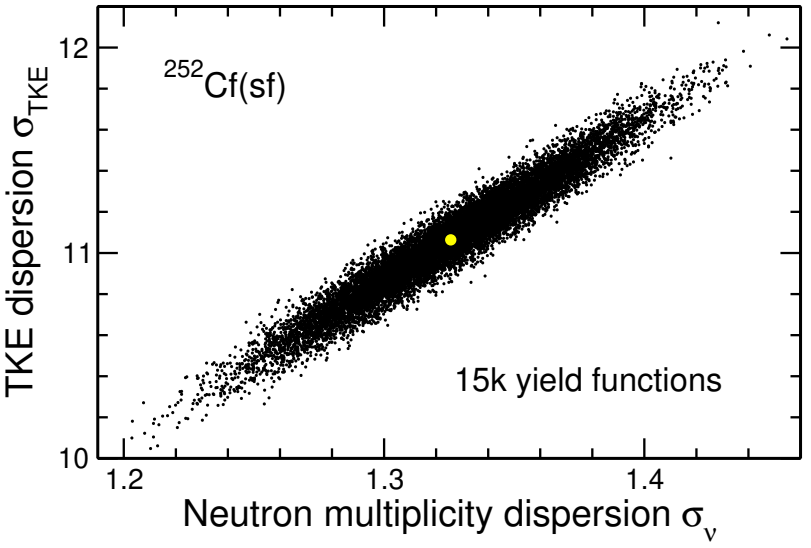

Figure 2. Scatter plot of $\sigma_{v}$ and $\sigma_{\overline{\mathrm{TKE}}}$, obtained for each of the 15,000 sampled yield functions. The overall average is indicated by the central dot. (From Ref. [6].)

tiplicity. Figure 1 shows a scatter plot of the values of $\left(\bar{v}^{(i)}, \overline{\mathrm{TKE}}^{(i)}\right)$ obtained for the full ensemble of 15,000 yield functions. It is clear that yields that give large/small values of $\bar{v}$ result in small/large mean TKE. Conversely, a similar 
scatter plot of the corresponding dispersions in $\bar{v}$ and $\overline{\mathrm{TKE}}$ show that large/small fluctuations in $\bar{v}$ are associated with large/small dispersions in TKE, see Fig. 2.

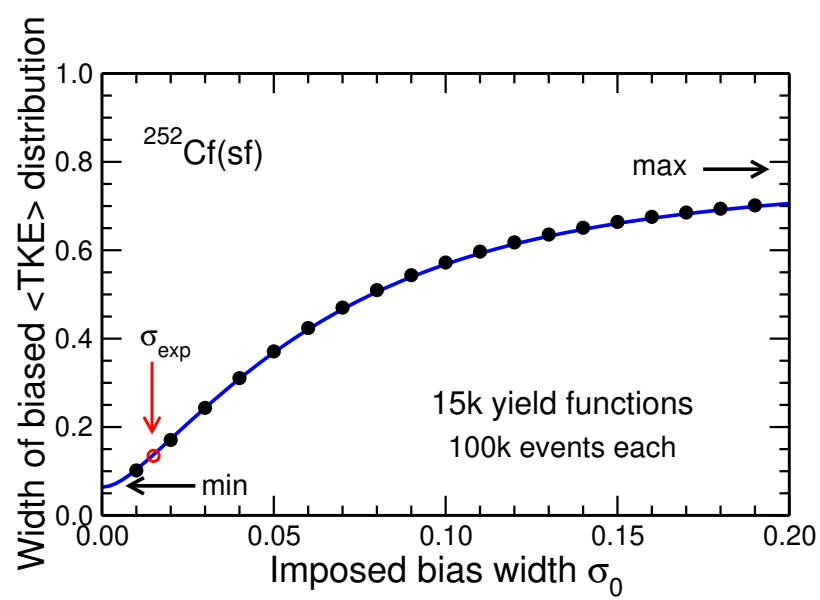

Figure 3. The width of the $\overline{\text { TKE }}$ distribution as a function of bias width $\sigma_{0}$ in the weight $W \sim \exp \left(-\delta v^{2} / 2 \sigma_{0}^{2}\right)$ of the individual yield functions $Y(A, Z, \mathrm{TKE})$, as extracted for specific values of $\sigma_{0}$ (dots) or as given in the analytical expression (9) (curve). The value obtained using $\sigma_{0}^{\text {eval }}$ is indicated by the circle. The calculations sampled 100,000 fission events from each of the 15,000 yield functions. (From Ref. [6].)

The correlation between $\bar{v}$ and $\overline{\text { TKE }}$ can be quantified by assuming that the joint distribution of these observables has a Gaussian form characterized by a covariance tensor. It was found that for the unbiased weights, $W_{i}=1 / N$, the dispersion on $\bar{v}$ calculated for the entire ensemble of yield functions, $\sigma_{0}=0.093$, was considerably larger than the evaluated dispersion, $\sigma_{0}^{\text {eval }}=0.015$. Thus the biased Gaussian weight as a function of $\bar{v}$ was introduced to preferentially select yield functions from the full ensemble to bring the variance on $\bar{v}$ closer to the evaluated one. The biased TKE variance is then

$$
\bar{\sigma}_{K}^{2}=\sigma_{K}^{2}-\frac{\sigma_{v K}^{2}}{\sigma_{v}^{2}+\sigma_{0}^{2}}
$$

where $\sigma_{K}^{2}=<(\overline{\mathrm{TKE}}-\prec \overline{\mathrm{TKE}}>)^{2}>, \sigma_{v}^{2}=<(\bar{v}-\prec \bar{v}>$ )$^{2}>$, and $\sigma_{v K}=\prec(\bar{v}-\prec \bar{v}>)(\overline{\mathrm{TKE}}-\prec \overline{\mathrm{TKE}}>)>$. In the limit of large bias width, $\sigma_{0} \rightarrow \infty$, labeled "max" in Fig. 3, $\bar{\sigma}_{K}^{2}$ is equivalent to the absence of an imposed bias, i.e. equal weights $\sim 1 / N)$, and $\bar{\sigma}_{K}^{2} \rightarrow \sigma_{K}^{2}$. For a very narrow bias width, $\sigma_{0} \rightarrow 0$, only yield functions where $\bar{v} \approx v_{0}$, thus $\left.\bar{\sigma}_{K}^{2} \rightarrow\left(\sigma_{v}^{2} \sigma_{K}^{2}-\sigma_{v K}^{2}\right) / \sigma_{v}^{2}\right)$, labeled "min" in Fig. 3. As seen in Fig. 3, the Gaussian approximation of the biased weight provides an excellent description of the result. For details of the derivation of $\bar{\sigma}_{K}^{2}$, see Ref. [6].

\section{Summary}

We have shown the results of an optimization of the FREYA parameters for spontaneous fission. We will further study optimization for neutron-induced fission. We have also shown that the neutron observables simulated by FREYA are rather insensitive to the initial yield functions. We also showed that there is a pronounced anticorrelation between $\bar{v}$ and $\overline{\text { TKE}}$. Indeed correlations exist among all observables that require a consistent treatment of fission.

Acknowledgments: This work was performed under the auspices of the U.S. Department of Energy under Contract numbers DE-AC52-07NA27344 (RV, LLNL), DE-AC02-05CH11231 (JR and LAB, LBNL), and DEAC52-06NA25396 (PT, LANL). The authors also thank the United States Department of Energy National Nuclear Security Administration Office of Defense Nuclear Nonproliferation Research and Development for support for this work.

\section{References}

[1] J. Randrup and R. Vogt, Phys. Rev. C 80, 024601 (2009).

[2] J.M. Verbeke, J. Randrup, and R. Vogt, Comp. Phys. Comm. 222, 263 (2018).

[3] J. Randrup and R. Vogt, Phys. Rev. C 89, 044601 (2014).

[4] R. Vogt and J. Randrup, Phys. Rev. C 84, 044621 (2011).

[5] J.T. Van Dyke, L.A. Bernstein, and R. Vogt, Nucl. Instrm. Meth. A 922, 36 (2019).

[6] J. Randrup, P. Talou, and R. Vogt, Phys. Rev. C 99, 054619 (2019).

[7] R. Capote, et al., Nucl. Data Sheets 110, 3107 (2009).

[8] R. Vogt, A. Nicholson, J. Randrup, I. Gauld, and S. Croft, LLNL-CONF-690741.

[9] S. Kirkpatrick, C.D. Gelatt, and M.P. Vecchi, Science 220, 4598 (1983).

[10] P. Santi and M. Miller, Nucl. Sci. Eng. 160, 190 (2008).

[11] C. Budtz-Jørgensen and H.-H. Knitter, Nucl. Phys. A 490, 307 (1988).

[12] F.-J. Hambsch and S. Oberstedt, Nucl. Phys. A 617, 347 (1997).

[13] E.M. Kozulin et al., Instrum. Exp. Tech. 51, 44 (2008).

[14] C. Romano, Y. Danon, R. Block, J. Thompson, E. Blain, and E. Bond, Phys. Rev. C 81, 014607 (2010).

[15] Sh. Zeynalov, F.-J. Hambsch, and S. Oberstedt, J. Korean Phys. Soc. 59, 1396 (2011).

[16] A. Göök, F.-J. Hambsch, and M. Vidali, Phys. Rev. C 90, 064611 (2014).

[17] S.L. Whetstone, Jr., Phys. Rev. 131, 1232 (1963).

[18] G.K. Mehta, J. Poitou, M. Ribrag, and C. Signarbieux, Phys. Rev. C 7, 373 (1973).

[19] A.C. Wahl, At. Data Nucl. Data Tables 39, 1 (1988).

[20] A.D. Carlson et al., Nucl. Data Sheets 148, 143 (2018). 